\title{
Generation and molecular characterisation of monoclonal IgG4 rheumatoid factor from a patient with rheumatoid arthritis
}

\author{
Masayuki Hakoda, Naoyuki Kamatani, Atsuo Taniguchi, Hiroyuki Ueda, \\ Hisashi Yamanaka, Chihiro Terai, Sadao Kashiwazaki
}

\begin{abstract}
Objective-To characterise IgG4 rheumatoid factor (RF) at the molecular level from a patient with rheumatoid arthritis. Methods-B cells were cloned from the peripheral blood of a patient with rheumatoid arthritis, using $E B$ virus transformation. The supernatants of the clones were screened for IgG RF activity by ELISA. Nucleotide sequences of the expressed immunoglobulin heavy and light chain genes of one IgG RF producing clone were determined by direct sequencing of the products of a polymerase chain reaction.

Results-One clone producing monospecific IgG4 RF was obtained. Sequence analysis of the heavy and light chain genes suggested the accumulation of somatic mutations resulting in amino acid replacement in complementarity determining regions.

Conclusions-The results may suggest an antigen driven response in the generation of IgG4 RF in rheumatoid arthritis disease processes.

(Ann Rheum Dis 1997;56:74-77)
\end{abstract}

Rheumatoid factor (RF), the autoantibody directed against the $\mathrm{Fc}$ portion of $\mathrm{IgG}$ molecules, has long been used as a diagnostic marker for rheumatoid arthritis. ${ }^{1}$ Evidence suggesting the pathogenic roles of RF in rheumatoid disease processes is strong, but other reports also point to physiological roles of RF in the clearance of immune complexes. ${ }^{2}$ To understand the induction mechanisms for RF in patients with rheumatoid arthritis, extensive sequence analyses have been performed on the immunoglobulin genes of RF producing B cell clones. To date, sequences have been obtained from more than 30 clones producing monospecific IgM RF. These studies suggest that in at least some instances an antigen driven affinity maturation process generates monospecific IgM RF producing clones.

$\mathrm{RF}$ of the IgG class are reportedly more pathogenic than IgM RF, since IgG RF potentially may self associate and form complexes which can cause complement consumption leading to chronic synovial inflammation. Thus an understanding of the mechanisms underlying the production of IgG RF in patients with rheumatoid arthritis may be relevant to clarifying the pathogenesis of the disease. We report here the generation of an IgG4 RF producing B cell clone, KRT-1, from the peripheral blood of a patient with rheumatoid arthritis. Although IgG4 is a minor component in the serum IgG in both patients with rheumatoid arthritis and normal individuals, this subclass and IgG1 reportedly share a major part in circulating IgG RF in rheumatoid patients. ${ }^{4}$ Molecular characterisation of the KRT-1 IgG RF suggests that somatic mutations resulting in amino acid replacements are induced at higher frequencies in the complementarity determining region (CDR), as compared to the framework region $(\mathrm{RF})$, of $\mathrm{V}$ region genes.

\section{Methods}

GENERATION OF A B CELL CLONE SECRETING HUMAN IgG RF

Peripheral blood was obtained from a patient diagnosed as having rheumatoid arthritis, according to the 1987 criteria established by the American College of Rheumatology. ${ }^{1}$ B cells were cloned by distributing EB virus infected mononuclear cells into the wells of 96-well plates, using the technique described in an earlier report..$^{5}$ The rheumatoid factor activity (reactivity to rabbit IgG or human IgG Fc fragment, or both) and the heavy and light chain isotypes of the culture supernatants were determined by enzyme linked immunosorbent assay (ELISA), as in our earlier report. ${ }^{5}$ Supernatants with IgG RF activity were further characterised by examining the reactivity to other antigens, including thyroglobulin, insulin, type II collagen, ssDNA, and tetanus toxoid, as described. ${ }^{5}$ The IgG subclass of monoclonal IgG RF was determined by ELISA, using peroxidase conjugated mouse monoclonal anti-human IgG1, -3, or -4 (Zymed Laboratories, San Francisco, CA, USA). For the detection of IgG2, mouse monoclonal antihuman IgG2 (Zymed) was used and then peroxidase conjugated sheep $\mathrm{F}\left(\mathrm{ab}^{\prime}\right) 2$ fragment to mouse IgG (Cappel, Durham, NC, USA) was added. 2,2'-Azino-di[3-ethyl-benzthiazoline sulphonate] (Kirkegaard \& Perry Laboratories, Gaithersburg, MD, USA) was used to detect peroxidase conjugated antibodies bound on the plates, and absorbance at $405 \mathrm{~nm}$ was measured.

INHIBITION STUDY

The approximate affinity of a monoclonal IgG RF was determined using the ELISA method described by Friguet et al. ${ }^{6}$ Various concentra-
Accepted for publication 17 October 1996 
A

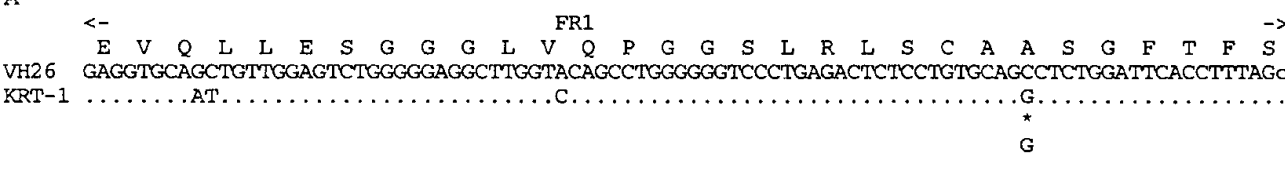

$<-\quad$ CDR1 $-><-\quad$ FR2 $\quad l->-\quad$ CDR2

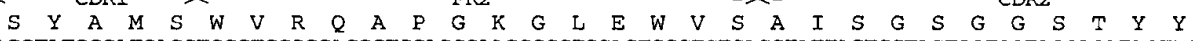
VH26 AGCTATGCCATGAGCTGGGTCCGCCAGGCTCCAGGGAAGGGGCTGGAGTGGGTCTCAGCTATTAGTGGTAGTGGTGGTAGCACATACTAC

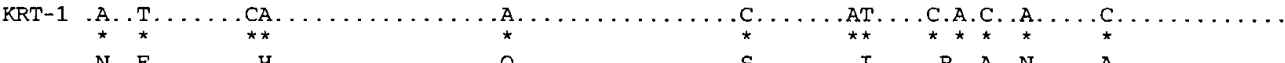

$\begin{array}{llllllllllllllllllllllllllllllllll}A R 3 & D & S & V & K & G & R & F & T & I & S & R & D & N & S & K & N & T & L & Y & L & Q & M & N & S & L & R & A & E & D\end{array}$ VH26 GCAGACTCCGTGAAGGGCCGGTTCACCATCTCCAGAGACAATTCCAAGAACACGCTGTATCTGCAAATGAACAGCCTGAGAGCCGAGGAC

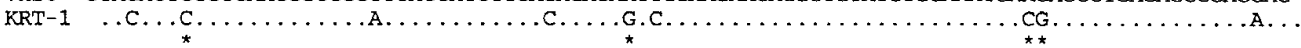

B

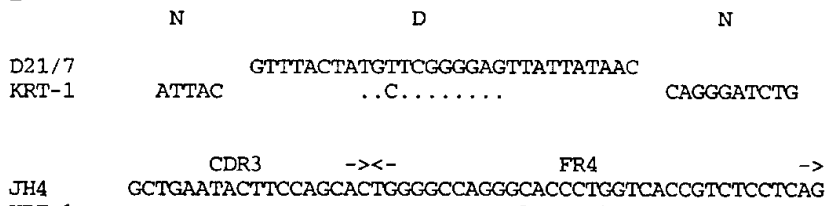

JH4 GCTGAATACTTCCAGCACTGGGGCCAGGGCACCCTGGTCACCGTCTCCTCAG

Figure 1 (A) The nucleotide sequence of the VH gene segment of KRT-1 compared with the sequence of the closest germline gene VH26. The amino acid sequence of KRT-1 is given above the nucleotide sequence. The dots denote nucleotide sequence identity. The sequence differences resulting in amino acid replacements are indicated by * and the substituted amino acid sequences are given below. (B) The nucleotide sequence of the N-D-N and the $\mathcal{F H}$ region of KRT-1 compared with the closest germline $D$ and $\mathcal{F H}$ genes.

tions of human IgG $\mathrm{Fc}$ fragment were incubated with $600 \mathrm{ng} \mathrm{ml}^{-1}$ of monoclonal IgG $\mathrm{RF}$ at room temperature for 16 hours. To measure the amount of IgG RF unbound to liquid phase IgG $\mathrm{Fc}$, the mixture was transferred to the plates coated with human IgG $F c$ and the IgG RF bound to the solid phase IgG Fc was detected by peroxidase conjugated goat $F\left(a b^{\prime}\right) 2$ fragment to human IgG Fab.

DETECTION OF EXPRESSED VH AND VK GENE FAMILIES

Total cellular RNA was extracted from a cloned B cell line using RNAzolTM B (Biotex Laboratories, Houston, TX, USA). cDNA synthesis and PCR amplification were carried out using a GeneAmp RNA PCR kit (Takara Shuzo, Kyoto, Japan). For the determination of VH family usage, VH family specific leader sequences were used as $5^{\prime}$ primers as described. ${ }^{5}$ The $\mathrm{C} \gamma$ sequence (5'GTAGTCCTTGACCAGGCA 3') was used as the $3^{\prime}$ primer. For the determination of $\mathrm{V}_{K}$ gene usage, leader sequences of $\mathrm{V}_{K}$ I-IV were used as $5^{\prime}$ primers (VKI, II: 5'CTCAGCTCCTGGGGCTCCTG3'; VkIII: 5'CTCCTGCTACTCTGGCTG3'; VKIV: 5'ATGGTGTTGCAGACCCAGGT3') and $\mathrm{C}_{K}$ sequence (5'TCTAGATCATCAGATGGCGGGAA 3') was used as the $3^{\prime}$ primer. PCR conditions were $94^{\circ} \mathrm{C}$ for 40 seconds, $60^{\circ} \mathrm{C}$ for one minute, $72^{\circ} \mathrm{C}$ for one minute for 35 cycles, and for the last cycle the condition was $72^{\circ} \mathrm{C}$ for five minutes.
Mutations in the $V$ regions of KRT-1

\begin{tabular}{lllcc}
\hline \multirow{2}{*}{ Region } & \multirow{2}{*}{$\begin{array}{l}\text { No of } \\
\text { mutations }\end{array}$} & \multicolumn{3}{l}{ Nature of mutations } \\
\cline { 3 - 5 } & & Replacement & Silent & $R / S$ \\
\hline H chain & 14 & 13 & 1 & 13 \\
CDR & 18 & 7 & 11 & 0.64 \\
FR & & & & \\
L chain & 6 & 4 & 2 & 2 \\
CDR & 11 & 4 & 7 & 0.57 \\
FR & & & & \\
\hline
\end{tabular}

DIRECT SEQUENCING OF THE PCR PRODUCTS The PCR products were directly sequenced without cloning into a vector as described. ${ }^{7}$ The sequences obtained were translated into amino acid sequences by a self made computer program.

\section{Results}

One B cell clone designated KRT-1 secreting IgG4 RF was obtained from the peripheral blood of a rheumatoid arthritis patient after two rounds of cloning procedures. The KRT-1 antibody was reactive with rabbit IgG and human IgG Fc fragment but non-reactive with a panel of five other antigens; therefore it was considered to be monoreactive. The heavy and light chain isotypes of KRT-1 were determined by ELISA to be IgG4 and $K$, respectively. The affinity of KRT-1 for human IgG Fc was estimated by an inhibition study. The $\mathrm{K}_{\mathrm{D}}$ value of KRT-1 for human IgG Fc was calculated to be $1.0 \times 10^{-7}$, a value comparable to those of high affinity IgG RF of other subclasses. ${ }^{89}$

PCR products were obtained from the cDNA synthesised from KRT-1 RNA only 
when the $\mathrm{VH} 3$ primer or the $\mathrm{V}_{K} \mathrm{I}$ (II) primer was used as the 5' primer. The PCR products were purified and then directly sequenced. A homology search, using the GenBank database, revealed that the KRT-1 VH sequence was most homologous $(90.5 \%)$ to the germline sequence $\mathrm{VH} 26$ in the VH3 family (fig 1A). The sequence differences between KRT-1 VH and VH26 were clustered in the CDR (fig 1A). Thus 14 of 77 nucleotides $(18.2 \%)$ in CDR1 and CDR2 were different in the two sequences, while 18 of $269(6.9 \%)$ in the FR were different (table). Furthermore, 13 of 14 nucleotide differences in the CDR resulted in amino acid changes, while only seven of 18 in the FR resulted in such changes (table). Assuming these nucleotide changes are derived from somatic mutations, the ratio of replacement (R) mutations to silent (S) mutations is 13.0 in CDR and 0.5 in FR (table). A sequence search revealed that 10 nucleotides in CDR3 were identical to the core sequence of D21/7 germline D gene (Fig 1B). Among 37 nucleotides in the most 3' portion of the KRT-1 variable region sequence, 34 were identical to the germline $\mathrm{JH} 4$ gene (fig $1 \mathrm{~B}$ ).

The light chain V gene of KRT-1 showed highest homology $(94.4 \%)$ to the germline HK102 gene in the $\mathrm{V}_{K} \mathrm{I}$ family (fig 2). The $\mathrm{J}_{K} 1$ gene was used in germline configuration (fig 2). Although the R/S ratio in the CDR of the light chain $\mathrm{V}$ gene was lower than in the heavy chain CDR, it was still higher than the R/S ratios in FR (table).

\section{Discussion}

To understand the mechanism for the production of IgG RF in rheumatoid arthritis patients, we generated a B-cell clone (KRT-1) that pro-

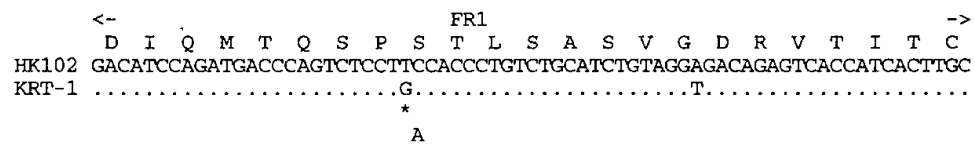

$\begin{array}{lllllllllllllllllllllllll}R & A & S & Q & Q & S & I & S & S & W & L & A & \text { W } & Y & Q & Q & K & P & G & K & A & A & P & K & L\end{array}$ HK102 CGGGCCAGTCAGAGTATTAGTAGCTGGTTGGCCTGGTATCAGCAGAAACCAGGGAAAGCCCCTAAGCTC

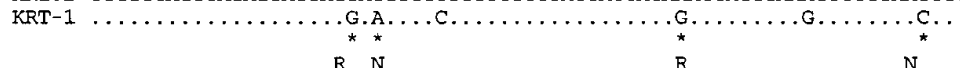

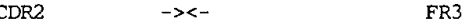

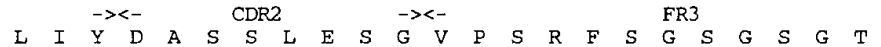

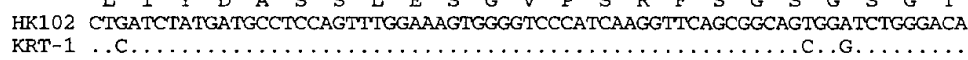
$\begin{array}{llllllllllllllllllllllllllllllllll}E & F & T & L & T & I & S & S & L & Q & P & D & D & F & A & T & Y & Y & C & Q & Q & Y & N\end{array}$ HK102 GAATTCACTCTCACCATCAGCAGCCTGCAGCCTGATGATTTTGCAACTTATTACTGCCAACAGTATAAT

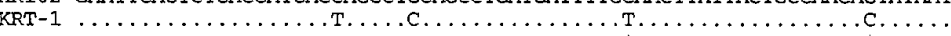
V

$\mathrm{H}$

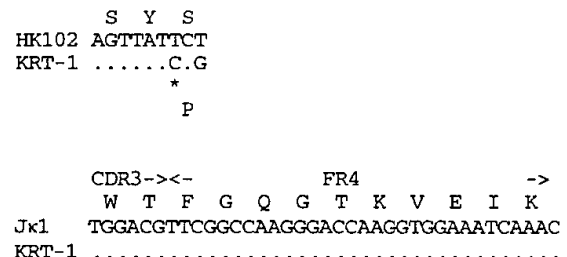

Figure 2 The light chain nucleotide sequence of KRT-1 compared with the closest germline $V_{K}$ and $\mathscr{f}_{K}$ genes. The amino acid sequence of KRT-1 is given above the nucleotide sequence. Dots denote nucleotide sequence identity. Sequence differences resulting in amino acid replacements are indicated by ${ }^{\star}$ and the substituted amino acid sequences are given below. duced a monoreactive IgG4 RF with high affinity. Although IgG4 is a minor component in the total serum IgG pool (approximately $5 \%$ ), it has been reported that the circulating IgG RF in rheumatoid patients is primarily composed of IgG1 and IgG4. ${ }^{4}$ There have been five reports describing the molecular characterisation of nine monoclonal IgG $\mathrm{RF}^{8-}$ 12; among these rheumatoid factors, only one is of the IgG4 4 class. ${ }^{10}$

A homology search revealed that the KRT-1 VH gene had the highest homology $(90.5 \%)$ with germline gene VH26. Since the germline $\mathrm{VH}$ sequences of the individual from whom KRT-1 was derived were not determined, there of course remains a possibility that the KRT-1 $\mathrm{VH}$ sequence was derived from a germline gene as yet unidentified or from a highly polymorphic gene. Recently, we have determined the $\mathrm{VH}$ sequences of $27 \operatorname{IgM} \mathrm{B}$ cell lines expressing the genes of VH3 family, in which the VH26 gene is included. ${ }^{7}{ }^{13}$ Except for the two sequences which showed $92.4 \%$ and $94.2 \%$ homology, respectively, the other 24 IgM VH sequences had more than 96\% homology with known germline genes (18 sequences had more than $99 \%$ homology with known germline genes). ${ }^{713}$ As such, the differences between the KRT-1 VH sequence and the most homologous VH26 sequence are at least partly derived from somatic mutations.

Most recently, a contribution of the constant region of IgG4 to RF activity was reported. ${ }^{14}$ Thus it may be possible that the RF activity of KRT-1 is also attributed to the IgG4 constant region. IgG4 antibodies have been found to be induced by chronic antigenic stimulation. ${ }^{15}$ Prevalence of IgG4 subclass in IgG RF in rheumatoid arthritis patients may reflect chronic antigenic stimulation in the disease process. Accumulation of replacement mutations in the CDRs in KRT-1 may support such a notion. Since the complement activating capacity of IgG4 is weaker than other subclasses, the pathogenic role of IgG4 RF seems to be limited compared to RF of other IgG subclasses. Rather, the presence of IgG4 $\mathrm{RF}$ may be the result of the longstanding immunological abnormalities. Further investigation examining the relations between IgG4 $\mathrm{RF}$ and clinical features such as disease chronicity and response to treatment may help to reveal the pathogenic significance of IgG4 $\mathrm{RF}$ in rheumatoid arthritis.

We appreciate Ms S Kurumada for excellent technical assistance and Prof $M$ W Schein (Rockville, MD, USA) for reviewing the manuscript.

1 Arnett FC, Edwirthy SM, Bloch DA, McShane DJ, Fries JF, Cooper NM, et al. The American Rheumatism Association 1987 revised criteria for the classification of rheumatoid arthritis. Arthritis Rheum 1988;31:315-24.

2 Coulie PG, Van Snick JL. Rheumatoid factor (RF) production during anamestic immune responses in the mouse. III. Activation of RF precursor cells is induced by their interaction with immune complexes and carrier specific helper T cells. J Exp Med 1985;161:88-97.

3 Randen I, Brown D, Thompson KM, Hughes-Jones N, Pascual V, Victor K, et al. Clonally related IgM rheumatoid factors undergo affinity maturation in the rheumatoid synovial tissue. J Immunol 1992;148:3296-301.

4 Cohen PL, Cheek RL, Hadler JA, Yount WJ, Eisenberg RA. The subclass distribution of human IgG rheumatoid The subclass distribution of human

5 Hakoda $M$, Ishimoto $T$, Hashimoto $S$, Inoue $\mathrm{K}$, Taniguchi A, Kamatani N, et al. Selective infiltration of $B$ cells 
committed to the production of monoreactive rheumatoid factor in synovial tissue of patients with rheumatoid thritis Clin Immunol Immunopathol 1993;69:16-22.

6 Friguet B, Chaffotte AF, Djavadi-Ohaniance L, Goldberg ME. Measurement of the true affinity constant in solution of antigen-antibody complexes by enzyme-linked immunosorbent assay. J Immunol Methods 1985;77:305-19.

7 Hakoda M, Hayashimoto S, Yamanaka H, Terai C, Kamatani N, Kashiwazaki S. Molecular basis for the interaction between human IgM and staphylococcal protein A Clin Immunol Immunopathol 1994;72:394-401.

8 Lu EW, Deftos M, Olee T, Huang D-F, Soto-Gil RW, Carson DA, et al. Generation and molecular analyses of two rheumatoid synovial fluid-derived IgG rheumatoid factors. Arthritis Rheum 1993;36:927-37.

9 Ezaki I, Shingu $M$, Hashimoto $M$, Isayama T, Tohmatsu J, Kanda $\mathrm{H}$, et al. Analysis of the genes encoding the variable regions of human IgG rheumatoid factor. J Rheumatol regions of human

10 Weisbart RH, Wong AL, Noritake D, Kacena A, Chan G, Ruland $\mathrm{C}$, et al. The rheumatoid factor reactivity of a
human IgG monoclonal autoantibody is encoded by a human IgG monoclonal autoantibody is encoded by a variant 801 .
11 Olee T, Lu EW, Huang D-F, Soto-Gil RW, Deftos M, Kozin $\mathrm{F}$, et al. Genetic analysis of self-associating immunoglobulin $\mathrm{G}$ rheumatoid factors from two rheumatoid synovia implicates an antigen-driven response. J Exp Med 1992;175:831-42.

12 Randen I, Pascual V, Victor K, Thompson KM, Forre O, Capra JD, et al. Synovial IgG rheumatoid factors show evidence of an antigen-driven immune response and a shift in the V gene repertoire compared to IgM rheumatoid facin the $\mathrm{V}$ gene repertoire compared to Ig
tors. Eur J Immunol 1993;23:1220-5.

13 Hakoda M, Kamatani N, Hayashimoto-Kurumada S, Silverman GJ, Yamanaka H, Terai C, Kashiwazaki S. Differential binding avidities of human IgM for staphylococcal protein A derive from specific germ-line VH3 gene usage. J Immunol 1996;157:2976-81.

14 Zack DJ, Stempniak M, Wong AL, Weisbart RH. Localization of an Fc-binding reactivity to the constant region of human IgG4. J Immunol 1995; 155:5057-63.

15 Aalberse RC, van der Gaag R, van Leeuwen J. Serologic aspects of IgG4 antibodies. I. Prolonged immunization results in an IgG4-restricted response. J Immunol 1983;130:722-6.

\title{
Unusual but memorable
}

\author{
Series editor: Gary D Wright
}

This 45 year old woman with systemic lupus erythematosus developed painful subcutaneous swelling of her left hidfoot. The lesion eventually healed with resulting marked loss of subcutaneous tissue (figure). Panniculitis is uncommon in lupus. Its frequency is approximately $2-3 \%$ and it may develop before, with, or after the onset of discoid or systemic lupus. Frequent sites of involvement include the arms, buttocks, head, neck, and thighs. Typical presentation is of deep subcutaneous nodules or plaques with superimposed epidermal changes of atrophy, ulceration,

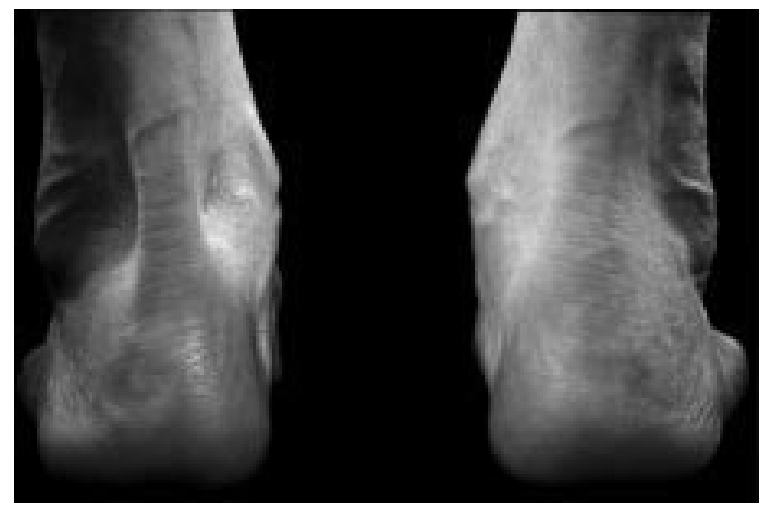
erythema, or poikiloderma. Histopathology reveals hyaline necrosis of fat and lymphocytic infiltrate around blood vessels and subcutaneous tissues. ${ }^{1}$ Calcification may be noted in old established lesions. The panniculitis tends to follow a chronic course with recurrences and remissions; resolution is commonly accompanied by large areas of depression and lipoatrophy. ${ }^{2}$

1 Sanchez NP, Peters MS, Winkleman RK. The histopathology of lupus erythematosus panniculitis. J Am Acad Dermatol $1981 ; 5: 673-80$.

2 Peters MS, Su WPD. Lupus erythematosus panniculitis. Med Clin North Am 1989;73:1113- 26.

Contributors: G D Wright, R POWELL, M DOHERTy. City Hospital, Nottingham NG5 1PB, UK. 